\title{
Joint image reconstruction and nonrigid motion estimation with a simple penalty that encourages local invertibility
}

\author{
Se Young Chun and Jeffrey A. Fessler \\ Department of Electrical Engineering and Computer Science, \\ The University of Michigan, Ann Arbor, MI
}

\begin{abstract}
Motion artifacts are a significant issue in medical image reconstruction. There are many methods for incorporating motion information into image reconstruction. However, there are fewer studies that focus on deformation regularization in motioncompensated image reconstruction. The usual choice for deformation regularization has been penalty functions based on the assumption that tissues are elastic. In the image registration field, there have been some methods proposed that impose deformation invertibility using constraints or regularization, assuming that organ motions are invertible transformations. However, most of these methods require very high memory or computation complexity, making them poorly suited for dealing with multiple images simultaneously in motion-compensated image reconstruction. Recently we proposed an image registration method that uses a simple penalty function based on a sufficient condition for the local invertibility of deformations. ${ }^{1}$ That approach encourages local invertibility in a fast and memory-efficient way. This paper investigates the use of that regularization method for the more challenging problem of joint image reconstruction and nonrigid motion estimation. A 2D PET simulation (based on realistic motion from real patient CT data) demonstrates the benefits of such motion regularization for joint image reconstruction/registration.
\end{abstract}

Keywords: motion-compensated image reconstruction, statistical image reconstruction, PET, topology preserving deformation, B-spline, joint estimation, alternating minimization

\section{INTRODUCTION}

Medical imaging modalities such as PET, SPECT, CT and MRI provide useful patient image information for diagnosis, treatment planning and intervention in clinical setting. However, due to their innate acquisition time limitations, there are some trade-offs between spatial resolution and motion artifact. Gating methods based on breathing signals or ECG signals ${ }^{2}$ can provide a good spatial resolution with reduced motion artifacts, but they can also reduce SNR by discarding potentially useful data.

There has been research to exploit all collected data and all motion information at the same time to get a better SNR. Most of the methods adopted nonrigid motion information to plug into their models since human organ motions are nonrigid. Motion information can be estimated separately, ${ }^{3-7}$ or simultaneously. ${ }^{8-10}$ Most research has focused on image reconstruction, whereas there has been less attention to motion estimation. In most of these methods the common choice for motion regularization is a simple quadratic roughness penalty or an elastic deformation penalty.

Regularizing or enforcing motion prior knowledge have been important issues in nonrigid image registration ${ }^{11}$ due to its ill-posedness. Motion invertibility or topology-preserving constraints have been one of the most reasonable motion prior and many have been proposing different methods to enforce it on nonrigid image registration problem. ${ }^{12,13}$ Unfortunately most such methods are hard to use for motion compensated image reconstruction because they require high computational cost and/or lots of memory space. Nevertheless, ensuring motion invertibility in motion-compensated image reconstruction is desirable. ${ }^{10}$

This paper proposes to use a simple penalty that encourages local invertibility ${ }^{1}$ in a motion-compensated image reconstruction framework. This penalty method is fast and memory efficient so it is well suited to motion-compensated image reconstruction.

This work was supported in part by NIH/NCI grant 1P01 CA87634.

Se Young Chun: E-mail: delight@umich.edu, Telephone: 7346155735

Jeffrey A. Fessler: fessler@umich.edu, Telephone: 7347631434

Medical Imaging 2009: Physics of Medical Imaging, edited by Ehsan Samei, Jiang Hsieh,

Proc. of SPIE Vol. 7258, $72580 \mathrm{U} \cdot$ ( ) 2009 SPIE

CCC code: $1605-7422 / 09 / \$ 18 \cdot$ doi: $10.1117 / 12.811067$

Proc. of SPIE Vol. 7258 72580U-1 


\section{METHOD}

\subsection{Measurement model}

Let $t_{m}$ denote the time of the $m$ th scan, i.e., the $m$ th frame in a dynamic study or the $m$ th gate in a gated study. We assume that the measurements are related to the object $\boldsymbol{f}_{m}=\left\{f\left(\cdot, t_{m}\right)\right\}$ as follows:

$$
\boldsymbol{y}_{m}=\boldsymbol{G} \boldsymbol{f}_{m}+\boldsymbol{\epsilon}_{m},
$$

where $\left\{\boldsymbol{y}_{1}, \ldots, \boldsymbol{y}_{M}\right\}$ denotes the projection measurements, $\boldsymbol{G}$ denotes the system model and $\boldsymbol{\epsilon}_{m}$ denotes noise. We assume that $\boldsymbol{f}_{m}$ is motion-free, i.e., the measurement $\boldsymbol{y}_{m}$ is motion-free, meaning that the object does not move during the $m$ th scan.

\subsection{Image reconstruction dealing with motion indirectly}

The goal is to reconstruct the 3D object $f$ from the data $\left\{\boldsymbol{y}_{1}, \ldots, \boldsymbol{y}_{M}\right\}$. Generally, using more measurement leads having higher spatial resolution or high SNR in medical imaging reconstruction. However, it can also lead to more motion artifacts. There are two extreme ways to reconstruct the object $f$. One is to reconstruct a low spatial resolution (low quality), but motion-free image. The other is to reconstruct a high spatial resolution (high quality), but motion-blurred image.

\subsubsection{Motion-free image reconstruction}

Since we assume that $\left\{\boldsymbol{y}_{1}, \ldots, \boldsymbol{y}_{M}\right\}$ do not contain any motion artifact, each reconstructed image $\hat{\boldsymbol{f}}_{m}$ from each collected data $\boldsymbol{y}_{m}$ will be motion-free. i.e.,

$$
\hat{\boldsymbol{f}}_{m}=\underset{\boldsymbol{f}_{m}}{\operatorname{argmin}} L\left(\boldsymbol{y}_{m}, \boldsymbol{G} \boldsymbol{f}_{m}\right)+\eta_{s} R_{s}\left(\boldsymbol{f}_{m}\right),
$$

where $L$ is a data fitting term (typically a negative log-likelihood) that quantifies how well the predicted projection data $\boldsymbol{G} \boldsymbol{f}_{m}$ matches the measurements $\boldsymbol{y}_{m} . R_{s}$ is an appropriate spatial regularizer and $\eta_{s}$ is a regularization parameter. Usually in medical imaging $\hat{\boldsymbol{f}}_{m}$ has suboptimal quality since we use only a small amount of data measurement $\boldsymbol{y}_{m}$.

\subsubsection{Motion-ignoring image reconstruction}

The other extreme is to use all measurements $\boldsymbol{y}_{A}=\left[\boldsymbol{y}_{1}, \cdots, \boldsymbol{y}_{M}\right]^{\prime}$ for reconstructing a single image $\boldsymbol{f}_{A}$, i.e., ignoring all motions:

$$
\hat{\boldsymbol{f}}_{A}=\underset{\boldsymbol{f}_{A}}{\operatorname{argmin}} L\left(\boldsymbol{y}_{A}, \boldsymbol{G}_{A} \boldsymbol{f}_{A}\right)+\eta_{A} R_{A}\left(\boldsymbol{f}_{A}\right),
$$

where $\boldsymbol{G}_{A}=[\boldsymbol{G}, \cdots, \boldsymbol{G}]^{\prime}$. Even though $\hat{f}_{A}$ generally has less noise, it will have severe motion artifacts, e.g. respiratory motion or cardiac motion in medical imaging.

\subsection{Image reconstruction dealing with motion directly}

We can use motion models to compensate for motion in image reconstruction directly. We can estimate motion information from $\boldsymbol{y}_{A}$ or from another data set. There are several ways to incorporate this motion information, but we focus only on two different approaches in this paper.

\subsubsection{Framewise image reconstruction}

One intuitive way is to combine all reconstructed images from (2) with motion compensation. The usual approach for motion compensation is to:

(i) reconstruct an image $\hat{\boldsymbol{f}}_{m}$ from the data $\boldsymbol{y}_{m}$ for each frame separately as in (2),

(ii) align each reconstructed image $\hat{f}_{m}$ to $\hat{f}_{1}$ by using a nonrigid image registration method and extract motion information $\hat{\boldsymbol{T}}_{m}^{-1}$, and

(iii) average the resulting images, i.e.,

$$
\hat{\boldsymbol{f}}_{1}^{\mathrm{FW}}=\frac{1}{M} \sum_{m=1}^{M} \hat{\boldsymbol{T}}_{m}^{-1} \hat{\boldsymbol{f}}_{m},
$$

where $\hat{\boldsymbol{T}}_{m}^{-1}$ is the transformation estimated for aligning $\hat{\boldsymbol{f}}_{m}$ to $\hat{\boldsymbol{f}}_{1}$. We refer to this approach as the framewise (FW) reconstruction method. One drawback of this approach is that in gated studies the SNR of the data for each frame is usually poor and this leads to poor image quality for each reconstructed frame $\hat{\boldsymbol{f}}_{m}$. These low quality images might in turn lead to inaccurate motion estimates. 


\subsubsection{Parametric motion model}

Another way to exploit motion information is to incorporate motion information into one model. In this paper, we introduce a parametric motion model,${ }^{8}$ a.k.a. joint reconstruction and registration model.

For our joint estimation approach, we assume that object $\boldsymbol{f}_{m}$ during the $m$ th frame is simply a warped version of an initial object under a motion model:

$$
\boldsymbol{f}_{m}=\boldsymbol{T}_{m} \boldsymbol{f}_{1}, \quad m=1, \ldots, M,
$$

where $\boldsymbol{T}_{m}$ is a spatial transformation that matches the two images $\boldsymbol{f}_{1}$ and $\boldsymbol{f}_{m}$. Then, our measurement model (1) will now contain only the image $\boldsymbol{f}_{1}$ instead of the all images $\boldsymbol{f}_{m}$ :

$$
\boldsymbol{y}_{A}=\boldsymbol{G}_{D} \boldsymbol{T}_{A} \boldsymbol{f}_{1}+\boldsymbol{\epsilon}_{A},
$$

where $\boldsymbol{G}_{D}=\operatorname{diag}\{\boldsymbol{G}, \cdots, \boldsymbol{G}\}, \boldsymbol{T}_{A}=\left[\boldsymbol{T}_{1}^{\prime}, \cdots, \boldsymbol{T}_{M}^{\prime}\right]^{\prime}$, and $\boldsymbol{\epsilon}_{A}=\left[\boldsymbol{\epsilon}_{1}^{\prime}, \cdots, \boldsymbol{\epsilon}_{M}^{\prime}\right]^{\prime}$. Using this model, we jointly estimate an image $\boldsymbol{f}_{1}$ and deformations $\boldsymbol{T}_{A}$ using the following type of cost function:

$$
\left(\hat{\boldsymbol{f}}_{1}, \hat{\boldsymbol{T}}_{2}, \ldots, \hat{\boldsymbol{T}}_{M}\right)=\underset{\boldsymbol{f}_{1}, \boldsymbol{T}_{2}, \ldots, \boldsymbol{T}_{M}}{\operatorname{argmin}} L\left(\boldsymbol{y}_{A}, \boldsymbol{G}_{D} \boldsymbol{T}_{A} \boldsymbol{f}_{1}\right)+\eta_{s}^{\prime} R_{s}\left(\boldsymbol{f}_{1}\right)+\eta_{m}^{\prime} R_{m}\left(\boldsymbol{T}_{A}\right),
$$

where a function $R_{s}\left(\boldsymbol{f}_{1}\right)$ is a standard roughness regularizer of the image. $R_{m}\left(\boldsymbol{T}_{A}\right)$ is a regularizer for the deformation $\boldsymbol{T}_{A}$ where $\boldsymbol{T}_{1}$ is an identity matrix. $\eta_{s}^{\prime}$ and $\eta_{m}^{\prime}$ are regularization parameters for an image and motions. We minimize this cost function by alternating between updating the image and updating the deformation estimates.

\section{SPATIAL AND MOTION REGULARIZATION METHODS}

\subsection{Spatial regularizers that have the same spatial resolutions}

Determining appropriate spatial regularization penalties for both framewise image reconstruction method and parametric motion model is an important issue for fair comparison. To analyze this in detail, we focus on emission tomography. There has been research on finding the relationship between the spatial resolution and the spatial regularization penalty value for a quadratic penalty. ${ }^{14} \mathrm{We}$ adopt this method here due to its simplicity. Ahn et al. proposed a spatial resolution analysis for non-quadratic spatial penalty ${ }^{15}$

For weighted least square data fidelity term that we use in this paper, ${ }^{16}$ the local impulse response approximation for (2) is

$$
l^{j}=\left[\boldsymbol{G}^{\prime} \boldsymbol{W} \boldsymbol{G}+\eta_{s} \boldsymbol{R}_{s}\right]^{-1} \boldsymbol{G}^{\prime} \boldsymbol{W} \boldsymbol{G} e^{j}
$$

where $\boldsymbol{W}$ is a weight (usually $\operatorname{diag}\left\{\frac{1}{y}\right\}$ ) and $e^{j}$ is an impulse at the $j$ pixel. This local impulse response depends on a measurement $\boldsymbol{y}$ and it seems hard to determine $\eta_{s}$ in advance. However, this measurement term can be decoupled approximately and we can determine $\eta_{s}$ to have the same spatial resolution for any measurement:

$$
l^{j} \approx\left[\boldsymbol{G}^{\prime} \boldsymbol{G}+\eta_{s} / \kappa_{j}^{2} \boldsymbol{R}_{s}\right]^{-1} \boldsymbol{G}^{\prime} \boldsymbol{G} e^{j}
$$

where $\kappa_{j}^{2}=\sum_{i} g_{i j}^{2} w_{i} / \sum_{i} g_{i j}^{2}, g_{i j}=[\boldsymbol{G}]_{i j}$ and $w_{i}=[\boldsymbol{W}]_{i i}$. Now we can choose a measurement-independent spatial regularization parameter $\eta_{s}$ for a given desired spatial resolution (full width of half maximum).

Spatial resolution analysis for (7) is more difficult due to the motion term $\boldsymbol{T}$. For simplicity, we assume that each transformation $\boldsymbol{T}_{m}$ is an identity. Further analysis on the relationship between spatial resolution and motion will be a future work. Then, (9) will be changed into

$$
l^{j} \approx\left[\boldsymbol{G}^{\prime} \boldsymbol{G}+\eta_{s}^{\prime} / M / \kappa_{j}^{2} \boldsymbol{R}_{s}\right]^{-1} \boldsymbol{G}^{\prime} \boldsymbol{G} e^{j}
$$

and we need to set $\eta_{s}^{\prime}=M \eta_{s}$ to maintain the same spatial resolution. 


\subsection{Motion regularizers that encourage local invertibility}

It is hard to determine fair motion regularization parameter values $\left(\eta_{m}^{\prime}\right)$ for both framewise image reconstruction and parametric motion model since they do not use the same data fidelity term. However, the purpose of having a motion regularization term is to encourage the local invertibility of transformation $\boldsymbol{T}_{A}$. Therefore, we determined appropriate motion regularization parameters to achieve the closest data matching as well as all positive Jacobian determinant values of transformations on all image pixels empirically.

We consider B-spline based nonrigid transformation

$$
\underline{T}(\underline{r})=\underline{r}+\underline{d}(\underline{r}),
$$

where $\underline{r}=(x, y, z)$ and $\underline{d}(\underline{r})$ is the deformation. We model the 3D deformation $\underline{d}=\left(d^{x}, d^{y}, d^{z}\right)$ using a tensor product of $n$ th-order B-splines as follows:

$$
d^{q}(\underline{r})=\sum_{i, j, k} c_{i, j, k}^{q} \beta^{n}\left(\frac{x}{m_{x}}-i\right) \beta^{n}\left(\frac{y}{m_{y}}-j\right) \beta^{n}\left(\frac{z}{m_{z}}-k\right)
$$

where $q \in\{x, y, z\}$ and $\beta^{n}$ is a $n$ th-order B-spline basis. Then we can use a simple penalty that encourages the local invertibility of deformation. ${ }^{1,17}$ This penalty is based on the following sufficient condition.

THEOREM 1. Suppose $0 \leq k_{q}<\frac{1}{2}$ for $q \in\{x, y, z\}$. Define:

$$
\begin{aligned}
C_{4}=\{\underline{c}: & -m_{x} k_{x} \leq c_{i+1, j, k}^{x}-c_{i, j, k}^{x} \leq m_{x} K_{x}, \\
& -m_{y} k_{y} \leq c_{i, j+1, k}^{y}-c_{i, j, k}^{y} \leq m_{y} K_{y}, \\
& -m_{z} k_{z} \leq c_{i, j, k+1}^{z}-c_{i, j, k}^{z} \leq m_{z} K_{z}, \\
& \left|c_{i+1, j, k}^{q}-c_{i, j, k}^{q}\right| \leq m_{q} k_{q} \text { for } q=y, z, \\
& \left.\left|c_{i, j+1, k}^{q}-c_{i, j, k}^{q}\right| \leq m_{q} k_{q} \text { for } q=x, z, \quad \forall i, j, k\right\} . \\
& \left|c_{i, j, k+1}^{q}-c_{i, j, k}^{q}\right| \leq m_{q} k_{q} \text { for } q=x, y, \quad \forall i,
\end{aligned}
$$

In (12), if $\underline{c} \in C_{4}$ then the Jacobian determinant of $\underline{T},|\mathbf{J}|$, satisfies the bounds

$$
1-\left(k_{x}+k_{y}+k_{z}\right) \leq|\mathbf{J}| \leq\left(1+K_{x}\right)\left(1+K_{y}\right)\left(1+K_{z}\right)+\left(1+K_{x}\right) k_{y} k_{z}+k_{x}\left(1+K_{y}\right) k_{z}+k_{x} k_{y}\left(1+K_{z}\right)
$$

$\forall \underline{r} \in \mathbf{R}^{3}$. Moreover, if $k_{x}+k_{y}+k_{z}<1$, then the transformation (11) is locally invertible everywhere.

We discourage values outside this sufficient condition by using a penalty function:

$$
p\left(t ; \zeta_{1}, \zeta_{2}\right)= \begin{cases}\frac{1}{2}\left(t-\zeta_{1}\right)^{2}, & t \leq \zeta_{1} \\ 0, & \zeta_{1}<t \leq \zeta_{2} \\ \frac{1}{2}\left(t-\zeta_{2}\right)^{2}, & \text { otherwise }\end{cases}
$$

where $t$ is the difference between adjacent B-spline coefficients and $\zeta_{1}, \zeta_{2}$ are determined based on Theorem 1 .

This work is the first use of this regularization method for joint image reconstruction / deformation estimation. Most of methods using motion invertibility priors in B-spline motion transformation require B-spline interpolation for partial derivatives and these require lots of memory and computation. However, this proposed penalty deals with B-spline coefficients directly which are usually much fewer than image pixels. Since it deals with much less data and does not require much computation resource for B-spline interpolation, this type of penalty is very fast and memory efficient for regularizing non-invertible deformations. ${ }^{1,17}$ This aspect should be a great advantage when we deal with $3 \mathrm{D}$ volume data.

\section{RESULTS}

We simulated 2D PET scans for 5 respiratory gates (at 0,20, 40, 60 and 80\% inhalation) using reconstructed CT images from a real patient shown in Figure 1. In this way, we have a known ground truth for image intensity and also have realistic breathing motion. The original image has $256 \times 256$ image resolution respectively with $1.7 \mathrm{~mm}$ pixel size. We projected 


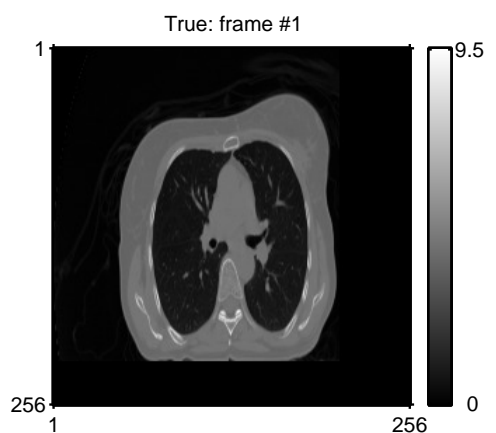

True: frame \#4

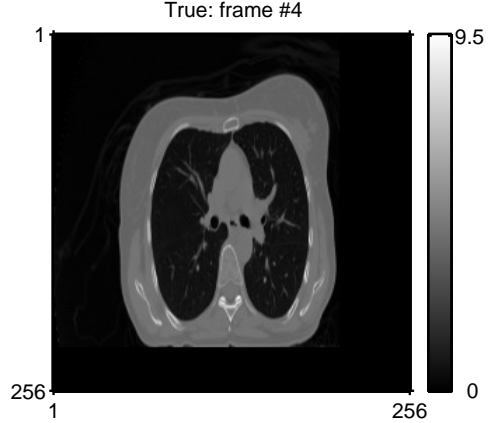

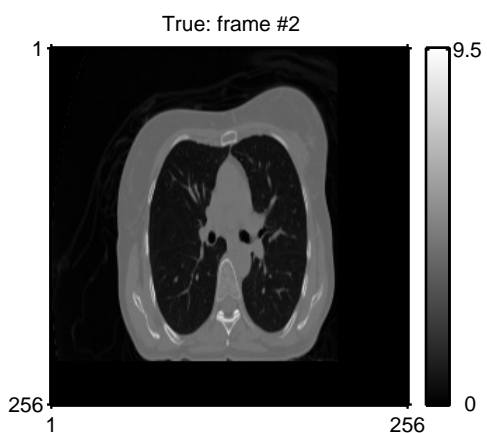

True: frame \#5

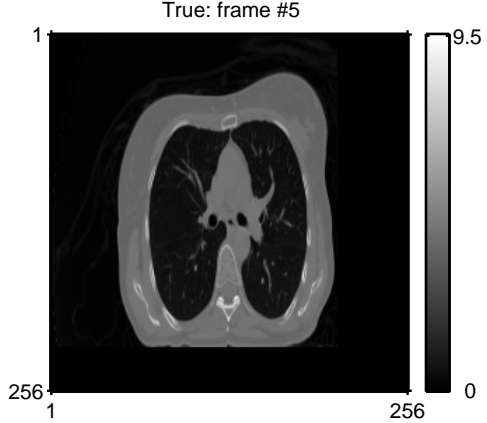

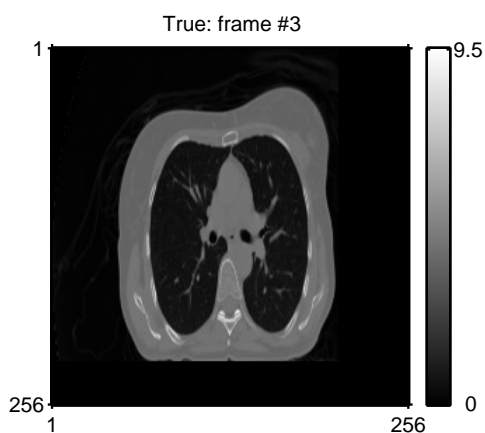

True: sum of all frames

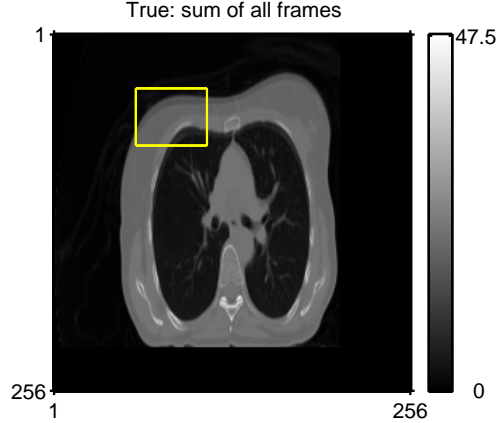

Figure 1. 2D images of a patient at 0, 20,40,60, and 80\% inhale (from breath-hold CT scans) and the sum of all images (last). Yellow box shows that there is a motion artifact due to breathing motion.

these original images by using CTI 931 PET scanner geometry with 160 detector resolution, $3.375 \mathrm{~mm}$ distance between detector cells, 192 angle views, $6.75 \mathrm{~mm}$ strip width and 10\% random coincidences. We used 200K mean true coincidences for each frame (1M total) and we ignored attenuation.

We reconstructed $128 \times 128$ images from these 5 sinograms. We used several methods: filtered back projection method (FBP) from each sinogram, penalized weighted least square (PWLS) ${ }^{16}$ from each sinogram and from the sum of all sinograms without motion correction, framewise image reconstruction (FW) with PWLS reconstructed images and nonrigid motion correction (knots are every 4 pixels), Penalized-Likelihood Consolidation (PLC) which uses parametric motion model with motion estimation from individualily reconstructed PWLS images, and parametric motion model based image reconstruction (PMM) ${ }^{8}$ For all reconstruction, we chose the regularization parameters to provide 2.7 pixels as a full width half maximum (FWHM). Conjugate gradient method was used for all optimization.

Figure 2 shows reconstructed images from image reconstruction schemes described above. Methods that use all sinograms (col 3, row 1 and all row 2 and 3) provide much better reconstructed results than methods that use a single sinogram (col 1 and 2, row 1). To see clear improvements in motion-compensated image reconstruction methods, Figure 3 shows a profile for PWLS from the sum of all sinograms, FW and PMM reconstruction images with proposed penalty.

For more quantitative analysis, Figure 4 shows normalized root mean square errors (NRMSE, \%) for each method with 20 realizations. Large NRMS error in PWLS reconstructed image from the sum of all sinograms is reduced with all motion correction methods. However, region of interest (ROI) area (yellow box in col 2, row 1 image) is not well corrected in quadratic penalty methods when we increase a regularization parameter value to have all positive Jacobian determinant values on all image pixels. This is because quadratic penalty seems to oversmooth deformations to satisfy invertibility property of deformation. ${ }^{1}$ Using more sinograms clearly reduces NRMS error compared to PWLS reconstruction from a single sinogram. However, most motion-compensated image reconstruction methods can not achieve NRMS error which PWLS reconstruction from the sum of all sinograms could achieve in the motionless region. In FW reconstructed images and PLC reconstructed images there are higher NRMS error areas near image edges. This is because estimated motion information from individually reconstructed PWLS images for each sinogram does not have an enough accuracy. It seems that PMM reconstructed images could correct some of these mismatches especially near the left and right side of outer edges of a patient. 


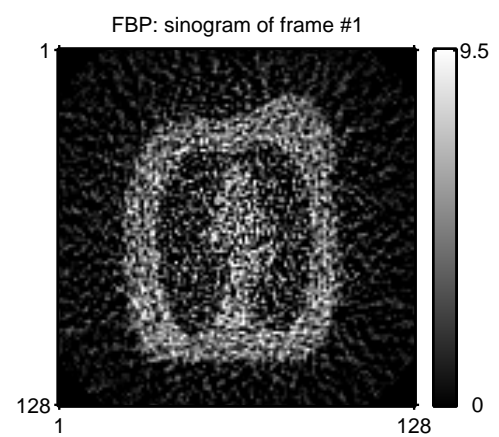

FW-PWLS: all sinograms, quadratic penalty

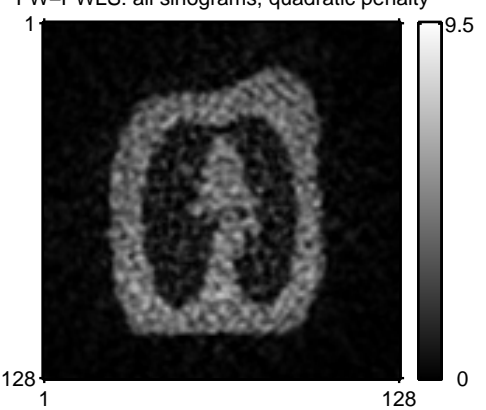

FW-PWLS: all sinograms, proposed penalty

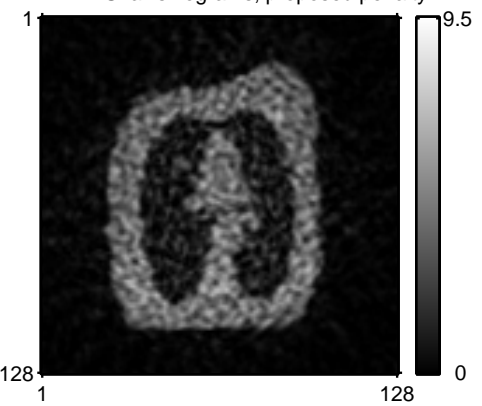

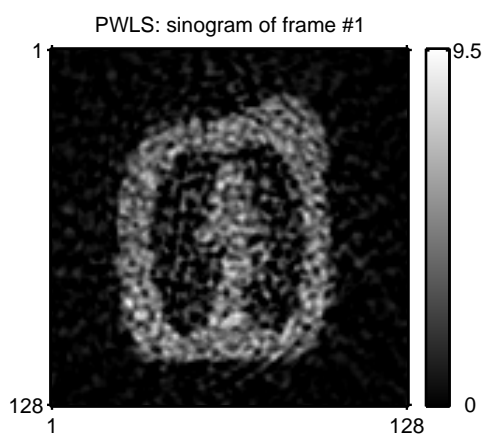

PLC-PWLS: all sinograms, quadratic penalty

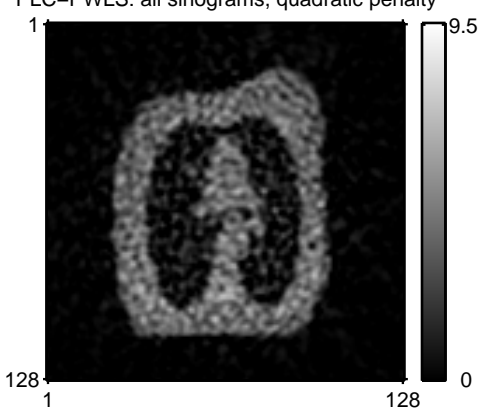

PLC-PWLS: all sinograms, proposed penalty

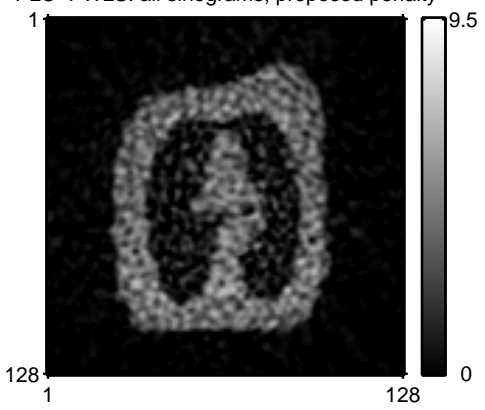

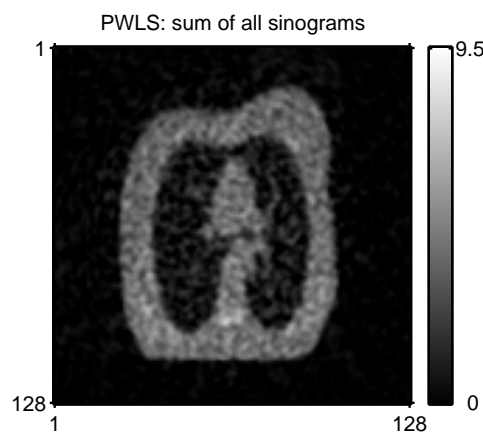

PMM-PWLS: all sinograms, quadratic penalty

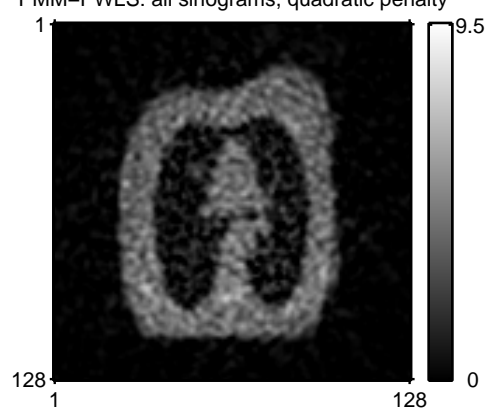

PMM-PWLS: all sinograms, proposed penalty

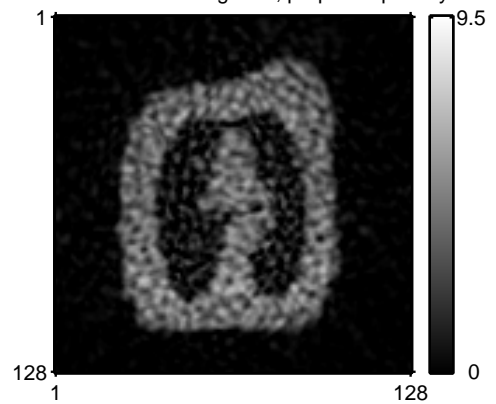

Figure 2. Reconstructed images from various image reconstruction methods: FBP ( 1 col, 1 row), PWLS ( 2 col, 1 row), PWLS with the sum of all sinograms ( 3 col, 1 row), FW with quadratic penalty $(1 \mathrm{col}, 2$ row $)$, PLC with quadratic penalty $(2 \mathrm{col}, 2$ row $)$, PMM with quadratic penalty ( $3 \mathrm{col}, 2 \mathrm{row})$, FW with proposed penalty (1 col, $3 \mathrm{row})$, PLC with proposed penalty ( $2 \mathrm{col}, 3 \mathrm{row})$, PMM with proposed penalty (3 col, 3 row).

Table 1. Mean and max NRMSE (\%) on all and ROI for each reconstruction method

\begin{tabular}{ccccc}
\hline \hline Method & mean NRMSE & max NRMSE & mean NRMSE of ROI & max NRMSE of ROI \\
\hline FBP: single frame & 14.9 & 50.1 & 28.7 & 42.6 \\
PWLS: single frame & 8.0 & 29.6 & 15.5 & 21.9 \\
PWLS: all frames & 4.4 & 45.5 & 12.6 & 31.7 \\
FW-PWLS, quadratic & 5.0 & 31.6 & 12.0 & 26.6 \\
FW-PWLS, proposed & 5.2 & 30.8 & 12.4 & 22.0 \\
PLC-PWLS, quadratic & 5.0 & 30.6 & 12.3 & 25.4 \\
PLC-PWLS, proposed & 5.3 & 30.6 & 12.6 & 21.1 \\
PMM-PWLS, quadratic & 4.6 & 38.2 & 11.8 & 28.7 \\
PMM-PWLS, proposed & 5.4 & 28.8 & 12.7 & 22.8 \\
\hline \hline
\end{tabular}


Profile: the effect of motion correction

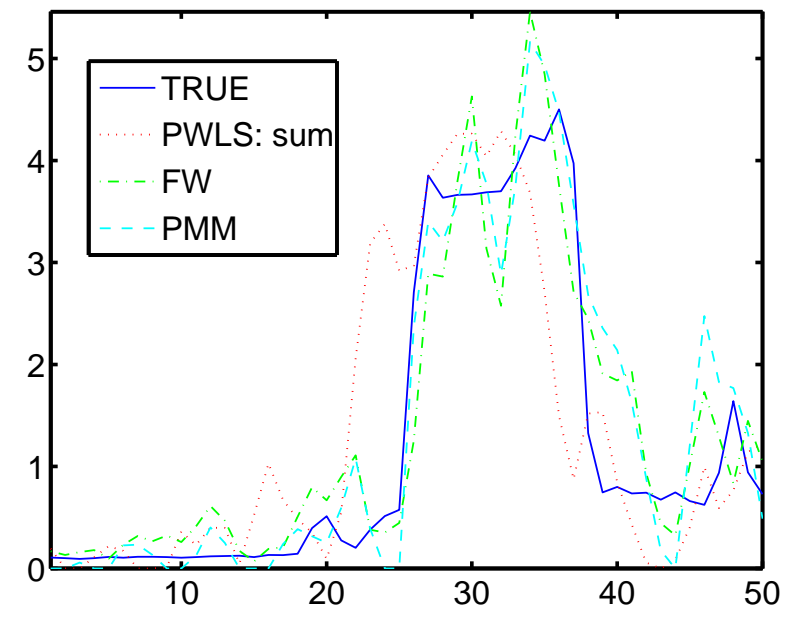

Figure 3. A profile of a reconstructed image from one realization. Motion-compensated methods (FW, PMM) corrected motion artifacts very well.

Table 1 shows mean and max NRMS errors for each method on the whole image domain and on ROI area which is indicated as a yellow box in Figure 4, col 3, row 1. Mean NRMS error is anywhere from 4.4\% to 5.4\% when we use all 5 sinograms regardless of motion correction. This is obviously much smaller than the case which uses a single sinogram $(8 \%)$. Since our simulated data does not contain much motion, PWLS reconstruction with the sum of all sinograms achieved the best mean NRMS error (4.4\%). However, this also has the largest max NRMS error (45.5\%) due to motion artifact in smaller region. This was reduced for all motion-compensated image reconstruction methods (28.8\% to $38.2 \%$ ). PMM reconstruction with our proposed method achieved the best max NRMS error $(28.8 \%)$ compared to max NRMS errors of other methods including FW methods. One interesting result is from PMM-PWLS, quadratic. It has the best mean NRMS error (4.6\%, close to $4.4 \%$ from PWLS: sum of all sinograms), but the worst max NRMS error (38.2\%). In this case, the estimated deformation looks almost flat so that it does not correct motions of organs inside a patient. Mean and max NRMS errors on ROI shows the clear difference between the results of quadratic and proposed penalties. Proposed penalty method can achive lower max NRMS error compared to quadratic penalty method for all types of reconstruction method. This is because our proposed method is based on more flexible structure compared to the smoothness penalty. ${ }^{1}$ The behavior of the PMM model will be an interesting future work.

Lastly, Figure 5 shows the warps of deformations generated by proposed and quadratic penalty methods. We increased a regularization parameter for each method until it achieves all Jacobian determinant values on all image pixels. Originally, almost $60 \%$ of image pixels had non-positive Jacobian determinant values. These deformation warps show that our proposed method has more flexibility for matching so that it may be able to achive a better image matching.

\section{DISCUSSION}

We introduced a motion regularizer based on an invertibility sufficient condition and incorporated it into for joint image reconstruction and nonrigid motion estimation. This paper showed that it is very important to exploit all information (sinograms) for better reconstructed images and motion compensation is crucial for correcting motion artifact. Our proposed penalty encourages the local invertibility of deformations ${ }^{1}$ and can be easily integrated into joint estimation scheme with modest computation. However, more study is needed to identify the characterstic of joint models and more comparisons should be performed with other motion penalties such as Jacobian determinant penalty.

\section{REFERENCES}

[1] Chun, S. Y. and Fessler, J. A., "A simple regularizer for B-spline nonrigid image registration that encourages local invertibility," IEEE J. Sel. Top. Sig. Proc. (2008). To appear.

[2] Nehmeh, S. A. and Erdi, Y. E., "Respiratory motion in positron emission tomography/computed tomography: a review," Semin Nucl Med. 38(3), 167-176 (2008). 


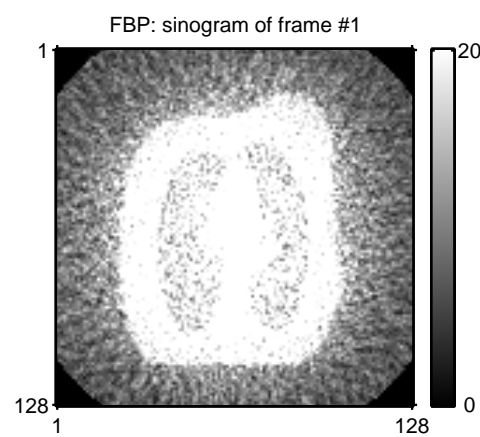

FW-PWLS: all sinograms, quadratic penalty

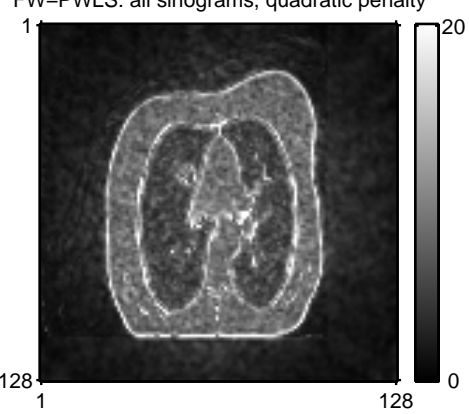

FW-PWLS: all sinograms, proposed penalty

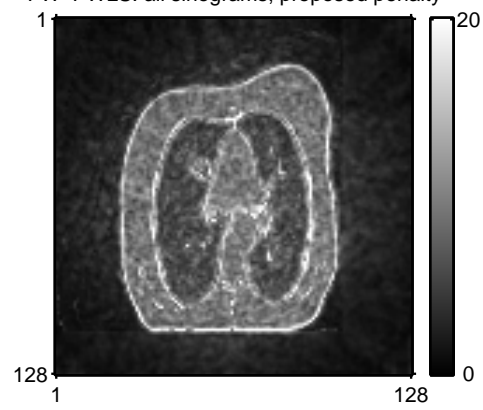

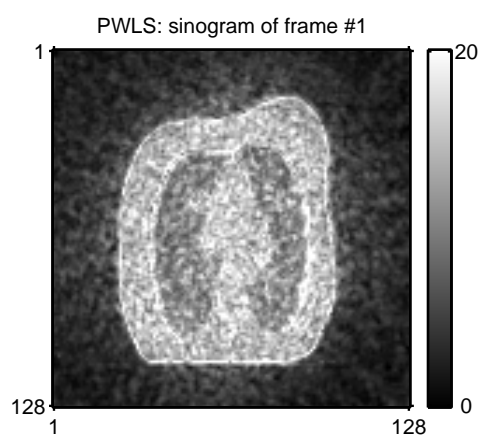

PLC-PWLS: all sinograms, quadratic penalty

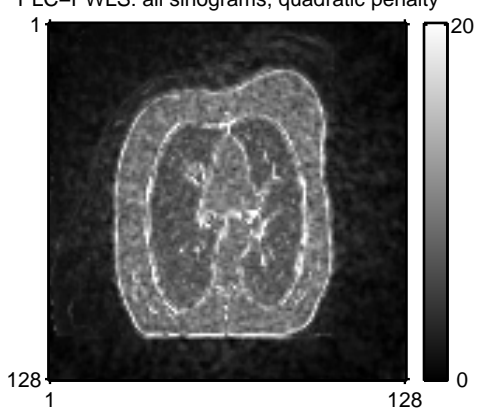

PLC-PWLS: all sinograms, proposed penalty

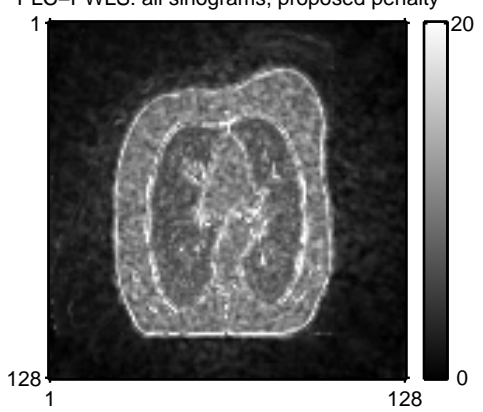

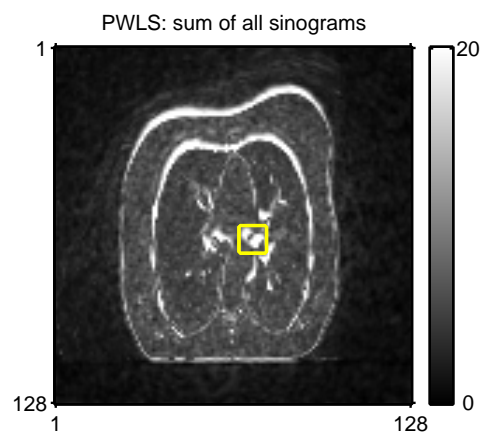

PMM-PWLS: all sinograms, quadratic penalty

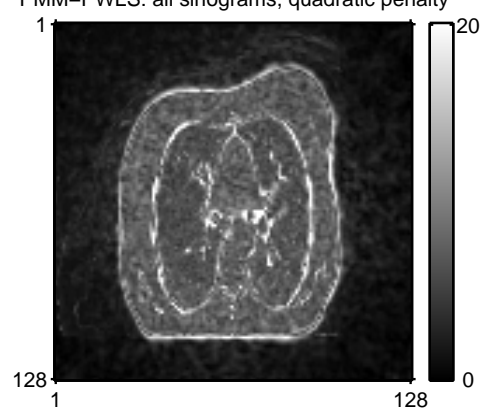

PMM-PWLS: all sinograms, proposed penalty

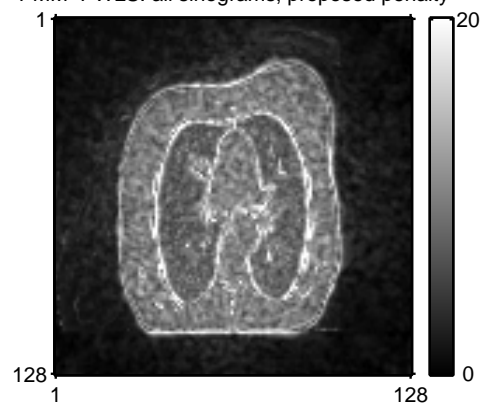

Figure 4. Normalized root mean square errors (NRMSE, \%) from various image reconstruction methods with 20 realizations: FBP (1 col, 1 row), PWLS (2 col, 1 row), PWLS with the sum of all sinograms ( 3 col, 1 row), FW with quadratic penalty (1 col, 2 row), PLC with quadratic penalty ( 2 col, 2 row), PMM with quadratic penalty (3 col, 2 row), FW with proposed penalty ( 1 col, 3 row), PLC with proposed penalty ( $2 \mathrm{col}, 3 \mathrm{row}$ ), PMM with proposed penalty (3 col, 3 row). We scaled NRMS errors to [0, 20\%] for better visualization. High NRMS errors due to motions reduced a lot through various motion correction scheme.

[3] Klein, G. J. and Huesman, R. H., "Four-dimensional processing of deformable cardiac PET data," Med Image Anal. 6(1), 29-46 (2002).

[4] Gravier, E. J., Yang, Y., King, M. A., and Jin, M., "Fully 4D motion-compensated reconstruction of cardiac SPECT images," Phys Med Biol. 51(18), 4603-4619 (2006).

[5] Qiao, F., Pan, T., Jr, J. W. C., and Mawlawi, O. R., "A motion-incorporated reconstruction method for gated PET studies," Physics in Medicine and Biology 51, 3769-3783 (August 2006).

[6] Li, T., Thorndyke, B., Schreibmann, E., Yang, Y., and Xing, L., "Model-based image reconstruction for four-dimensional PET," Medical Physics 33(5), 1288-1298 (2006).

[7] Lamare, F., Carbayo, M. J. L., Cresson, T., Kontaxakis, G., Santos, A., Rest, C. C. L., Reader, A. J., and Visvikis, D., "List-modebased reconstruction for respiratory motion correction in PET using non-rigid body transformations," Physics in Medicine and Biology 52, 5187-5204 (September 2007).

[8] Jacobson, M. W. and Fessler, J. A., "Joint estimation of image and deformation parameters in motion-corrected PET," in [Proc. IEEE Nuc. Sci. Symp. Med. Im. Conf.], 5, 3290-4 (2003).

[9] Mair, B. A., Gilland, D. R., and Sun, J., "Estimation of images and nonrigid deformations in gated emission CT," IEEE Trans. Med. Imag. 25, 1130-44 (Sept. 2006). 


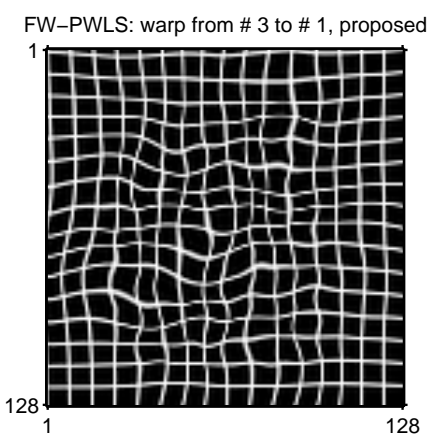

FW-PWLS: warp from \# 3 to \# 1, quadratic

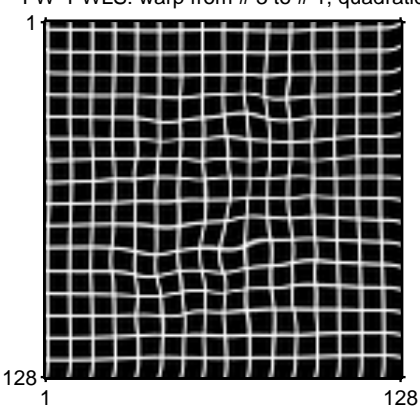

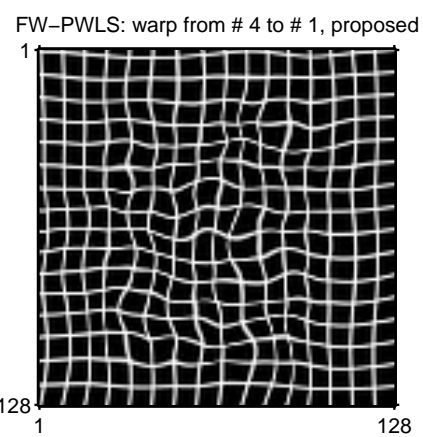

FW-PWLS: warp from \# 4 to \# 1, quadratic

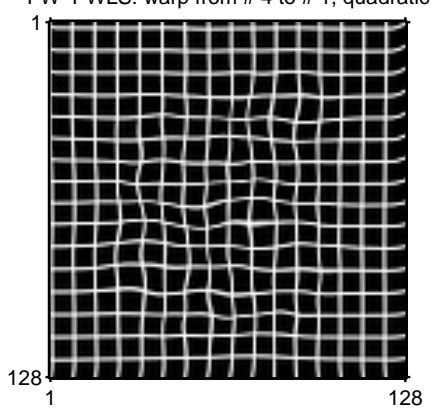

FW-PWLS: warp from \# 5 to \# 1, proposed

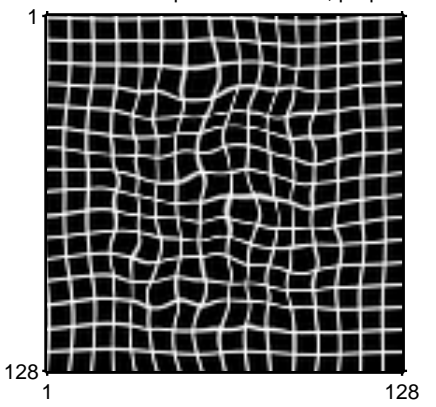

FW-PWLS: warp from \# 5 to \# 1, quadratic

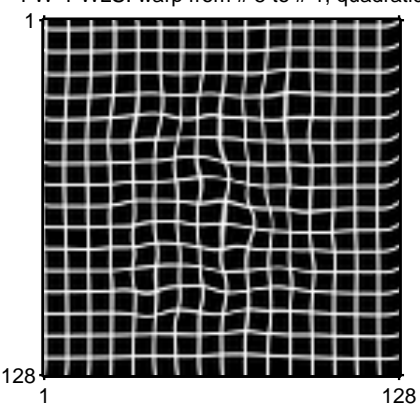

Figure 5. Deformations from frame 3 to frame 1, 4 to 1 , and 5 to 1 for each penalty method (proposed: row 1, quadratic: row 2). Our proposed penalty shows more flexibility for matching with positive Jacobian determinant values on all image pixels.

[10] Taguchi, K., Sun, Z., Segars, W. P., Fishman, E. K., and W.Tsui, B. M., "Image-domain motion compensated time resolved 4D cardiac CT," in [Proc. of SPIE, Medical Imaging], 6510 (2007).

[11] Crum, W. R., Hartkrns, T., and Hill, D. L. G., "Non-rigid image registration: theory and practice," The British Journal of Radiology 77, S140-S153 (2004).

[12] Noblet, V., Heinrich, C., Heitz, F., and Armspach, J., "3-D deformable image registration: A topology preservation scheme based on hierarchical deformation models and interval analysis optimization," IEEE Transactions on Image Processing 14(5), 553-566 (2005).

[13] Sdika, M., "A fast nonrigid image registration with constraints on the Jacobian using large scale constrained optimization," IEEE Transactions on Medical Imaging 27, 271-281 (February 2008).

[14] Fessler, J. A. and Rogers, W. L., "Spatial resolution properties of penalized-likelihood image reconstruction methods: Spaceinvariant tomographs," IEEE Trans. Im. Proc. 5, 1346-58 (Sept. 1996).

[15] Ahn, S. and Leahy, R. M., "Analysis of resolution and noise properties of nonquadratically regularized image reconstruction methods for PET," IEEE Trans. Med. Imag. 27, 413-24 (Mar. 2008).

[16] Fessler, J. A., "Penalized weighted least-squares image reconstruction for positron emission tomography," IEEE Transactions on Medical Imaging 13(2), 290-300 (1994).

[17] Chun, S. and Fessler, J. A., "Regularized methods for topology-preserving smooth nonrigid image registration using B-spline basis," in [Proc. IEEE Intl. Symp. Biomed. Imag. ], 1099-102 (2008). 